\title{
Challenges of Cardiopulmonary Resuscitation during Pregnancy
}

\author{
Padmaja Durga ${ }^{1}$ Shibani Padhy ${ }^{1}$ Anupama Bardaa \\ ${ }^{1}$ Anesthesiology and Intensive Care Nizam's Institute of Medical \\ Sciences, Panjagutta, Hyderabad, India \\ ${ }^{2}$ Medicine China Medical University, China \\ Ind J Car Dis Wom 2019;4:32-39
}

Address for correspondence Padmaja Durga, MD, DNB, Anesthesiology and Intensive Care, Nizam's Institute of Medical Sciences, Panjagutta 500082, Hyderabad, India (e-mail: padmajanims@yahoo.com).

\begin{abstract}
Keywords

- pregnancy

- cardiac arrest

- resuscitation

- perimortem cesarean section

Cardiac arrest, though rare, is the most feared complication in the pregnant woman as it involves two lives. Most arrests occur because of conditions that result from the pregnancy itself or from preexisting medical conditions exacerbated by the pregnancy. Prompt resuscitative efforts are crucial for favorable outcomes for the mother and fetus. The basic principles of resuscitation during pregnancy such as airway, breathing, and circulation are similar to the resuscitation in a cardiopulmonary arrest in any patient; however, certain modifications are necessary to account for the physiologic changes that occur during the pregnancy. Cardiopulmonary resuscitation (CPR) of the parturient should include uterine tilt or displacement to relieve the compression of the inferior vena cava and aorta by the gravid uterus, intubation using rapid sequence intubation with cricoid pressure, and timely perimortem cesarean section (PMCS). Ideally, the PMCS must be performed within 5 minutes of cardiac arrest if the pregnant woman does not have a return of spontaneous circulation, and resuscitation is deemed unsuccessful. The PMCS is performed if the gestational age is at least 20 weeks or the gravid uterus is evident. A high-quality CPR and multispecialty team approach, consisting of obstetricians, cardiologists, anesthesiologists, neonatologists, and nursing staff, is essential for survival.
\end{abstract}

\section{Introduction}

Cardiac arrest, though rare, is the most feared complication in the peripartum period. The incidence of cardiac arrest during pregnancy is 2.78 per 100,000 maternities, and mortality is about $42 \%{ }^{1}$ Resuscitation of the cardiac arrest in parturient aims at both the maternal and the fetal well-being. Survival rates are lower in the pregnant cardiac victim though younger than the patient with traditional cardiac arrest. The number of women with high risk attaining pregnancy is increasing, and so is the risk of possibility of cardiac arrests during pregnancy. ${ }^{2}$ There is an increasing need for improving the knowledge deficits, and resuscitation skills are among physicians for cardiac arrest during pregnancy. ${ }^{3}$

\section{Causes of Cardiac Arrest in Pregnancy}

The causes of cardiac arrest in a parturient are numerous (-Table 1). Most deaths result from acute conditions that are directly related or consequent to pregnancy. The cardiac arrests

DOI https://doi.org/ 10.1055/s-0039-1692304 also occur due to indirect causes resulting from worsening of preexisting comorbid conditions that could be further exacerbated by changes resulting from pregnancy. The majority of the serious cardiac complications of pregnancy occur in women not

Table 1 Causes of cardiac arrest during pregnancy

\begin{tabular}{|l|}
\hline Causes of pregnancy-related deaths \\
\hline Cardiovascular \\
Congenital heart disease \\
Ischemic heart disease \\
Valvular heart disease \\
Cardiomyopathy \\
Other acquired cardiac conditions \\
Noncardiovascular \\
Infection/sepsis \\
Hemorrhage \\
Thrombotic \\
Amniotic fluid embolism \\
Hypertensive \\
Cerebrovascular \\
Anesthetic causes \\
Others
\end{tabular}


recognized as having heart disease before pregnancy. About 25\% of cardiac arrests in pregnancy are related to anesthesia, which is potentially preventable. ${ }^{1,4}$ It is imperative for physicians to be able to diagnose and manage pregnancy-associated diseases and reversible causes of cardiac arrest in pregnancy.

\section{Cardiac Disease}

With an incidence on the rise, cardiac disease presently accounts for about 2.27 deaths for every 100,000 pregnant patients. Rheumatic heart disease continues to be the most common cardiac disease during pregnancy in India. ${ }^{5}$ There is an aggravation of underlying symptoms in patients with the rheumatic mitral disease during pregnancy. About 50\% of patients with mitral stenosis and $23 \%$ of mitral regurgitation decompensate during pregnancy and develop heart failure. ${ }^{6}$ In the United States, myocardial infarction and aortic dissection are the leading cardiac causes of maternal death. As compared with women of similar age, pregnant women have around three to four times higher risk of myocardial infarction. ${ }^{7}$ There are no known risk factors in most patients. The common causative factor is coronary artery dissection. The mechanisms are similar to aortic dissection. ${ }^{8}$ More women are now deferring pregnancy to a higher age that increases the chance of atherosclerosis-induced cardiac disease in parturients. ${ }^{9,10}$ Nearly $20 \%$ of women have intracoronary thrombus or arteriosclerosis on angiography. ${ }^{8}$ With fibrinolytics being relatively contraindicated, percutaneous coronary intervention $(\mathrm{PCI})$ remains the management strategy of choice for reperfusion in ST-elevation myocardial infarction in pregnancy. Advances in the medical field have now made it possible to witness many patients with congenital cardiac disease surviving into adulthood. It is, therefore, possible that they suffer a higher risk of cardiac events during pregnancy. ${ }^{11}$ Pulmonary hypertension, ${ }^{12}$ either primary or as a complication related to congenital heart disease, ${ }^{13}$ ranks third in the causes of maternal cardiac deaths. - Table 2 shows the high-risk conditions leading to cardiac morbidity and resultant maternal cardiac arrest. ${ }^{14}$

\section{Noncardiac Diseases}

- Eclampsia: Pregnancy-induced hypertension develops after 20 weeks, and eclampsia and multiorgan failure in severe cases complicate pregnancy. Maternal and fetal morbidity and mortality may result. ${ }^{15,16}$

- Pulmonary embolism (PE): Pregnancy is a hypercoagulable state, and it also aggravates the preexisting hypercoagulable conditions. PE is a significant complication in pregnancy. A high index of suspicion is required for diagnosis. Management of PE is according to the advanced cardiac life support (ACLS) guidelines. ${ }^{17}$ Massive PE is usually fatal though there have been few reports of successful use of fibrinolytics for massive, life-threatening PE. ${ }^{18}$

- Amniotic fluid embolism (AFE): The most feared and deadly cause of arrest in parturient is AFE. There are reports of high morbidity and mortality rates with the condition. ${ }^{19,20}$ The presentation of AFE is varied. Hypotension, bronchospasm, coagulopathy, fetal distress, and even cardiac arrest can occur. There are reports
Table 2 High-risk cardiac conditions during pregnancy

\begin{tabular}{l}
\hline High-risk cardiac conditions \\
\hline Myocardial infarction \\
Mechanical valve \\
Complex CHD \\
- Systemic RV \\
- Fontan circulation \\
Aortic dilatation \\
- Marfan's syndrome $40-45 \mathrm{~mm}$ \\
- Bicuspid AV 45-50 \\
Highest risk \\
- NYHA class III or IV \\
- Severe pulmonary hypertension \\
- Significant LV dysfunction \\
- Severe aortic or mitral stenosis \\
- Severe coarctation
\end{tabular}

Abbreviations: AV, aortic valve; CHD, congenital heart disease; LV, left ventricular; NYHA, New York Heart Association; RV, right ventricular.

of the use of cardiopulmonary bypass for successful management massive embolism in the peripartum period. ${ }^{21}$ Perimortem cesarean section (PMCS) can improve the chances of maternal and neonatal survival.

- Hemorrhagic shock: Hemorrhagic shock is an often encountered cause of cardiac arrest in the parturient. The placental pathologies such as abruptio placentae, placenta previa, or abnormalities of placental implantation pose a high risk for significant hemorrhage during labor. The other common causes of massive bleeding are surgical complications and persistent atonic uterus. Massive hemorrhage could culminate in cardiac arrest. ${ }^{22}$

- Iatrogenic: There are several preventable iatrogenic causes of cardiac arrest. They may be as simple as lack of proper instructions to avoid supine position. Patients may not have been instructed to return to their side after performing cervical examination or placement of monitoring devices in lithotomy or supine position. The venous return can decrease due to compression of the great vessels by gravid uterus, resulting in hypotension and possibly cardiac arrest, especially in the presence of epidural anesthesia.

Complications of therapy: Administration of tocolytic drugs, oxytocic drugs such as oxytocin, ergots, and medications such as magnesium is common in parturients. The adverse effects of these drugs can sometimes be fatal. Tocolytic drugs can cause cardiac rhythm disturbances, myocardial ischemia, and heart failure. ${ }^{23}$ There are reports of myocardial ischemia and consequent cardiac arrest with ergot derivatives in the parturient. The number of parturients with advanced age is increasing, and so would the coexisting medical problems. The presence of comorbidities may complicate treatment of premature labor with tocolysis or management of preeclampsia.

The iatrogenic overdose of magnesium can occur in patients receiving magnesium sulfate for treatment of eclampsia. Toxicity can develop even after low doses of magnesium if the woman becomes oliguric. Magnesium toxicity can result in cardiac effects. Electrocardiogram (ECG) interval changes such as prolonged conduction times resulting in PR, QRS, and QT interval prolongation 
occur at levels of magnesium between 2.5 and $5 \mathrm{mmol} / \mathrm{L}$. Further, an increase in magnesium levels to 6 to $10 \mathrm{mmol} / \mathrm{L}$ can cause atrioventricular (AV) nodal block, hemodynamic abnormalities, and cardiac arrest. Calcium administration can be helpful in reversing the effects of magnesium.

Complications of anesthesia: They are the important and preventable causes of cardiac arrest in labor and delivery. ${ }^{24}$ Local anesthetic toxicity can occur from drug overdosage. This complication is seen more often during prolonged labor when patients receive multiple top-up doses of local anesthetics. Occasionally, there can be complications even with regular dosing and testing. Intravascular migration of epidural catheters can lead to systemic toxicity, or migration into the subarachnoid space can cause total spinal anesthesia. Severe hypotension could result in hypovolemic patients receiving either regional or general anesthesia.

- Cardiac arrest from other etiology: Cardiac arrest can occur during pregnancy due to causes that are also seen in nonpregnant individuals such as trauma and anaphylaxis.

\section{Why Is Resuscitation in Parturient Different?}

Physiologic changes of pregnancy that influence cardiac arrest and resuscitation in a parturient: Compensatory physiologic changes occur in pregnancy to accommodate the needs for the growing fetus and uterus. These changes not only influence recommendations and guidelines for management of the pregnant cardiac arrest patient but also make the diagnosis and treatment difficult during emergencies. Management of such cases requires a complete understanding of these physiologic changes that occur in pregnancy. - Fig. 1 summarizes the implications of these physiologic changes.

Aortocaval compression in the supine position can decrease cardiac output by 30 to $40 \%$, resulting in supine hypotension syndrome. Supine position is often not tolerated after 30 weeks' gestation. It is therefore advised to maintain a position that produces the displacement of the uterus to the left, especially during the third trimester to relieve the aortocaval compression ( - Fig. 2 ).

The cardiac output increases by 1 to $1.5 \mathrm{~L} / \mathrm{min}$ after the 10th week of gestation. Maternal heart rate increases by 15 to 20 beats above nonpregnant values throughout pregnancy. Maternal blood pressure is lower than nonpregnant values by 10 to $15 \mathrm{~mm} \mathrm{Hg}$. Therefore, severe bleeding can occur without the usually seen physical signs of hypovolemia such as hypotension and tachycardia. Physiologic anemia with low hematocrit occurs at about 34 weeks' gestation due to an increase in plasma volume by 40 to $50 \%$ accompanied by a lesser increase in the volume of red blood cells. A lower

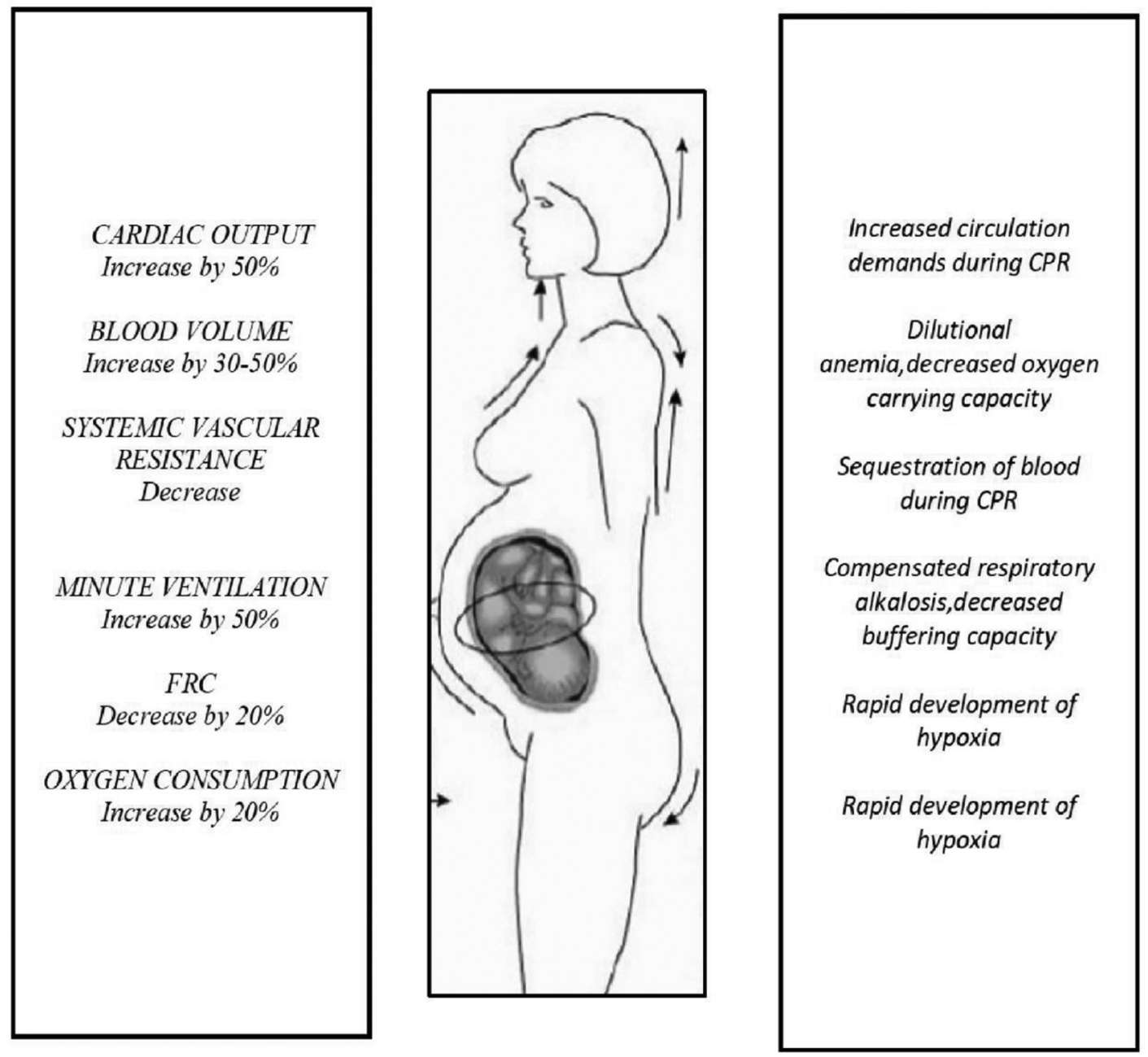

Fig. 1 Cardiorespiratory changes in pregnancy and their effects on resuscitation. CPR, cardiopulmonary resuscitation; FRC, functional residual capacity. 


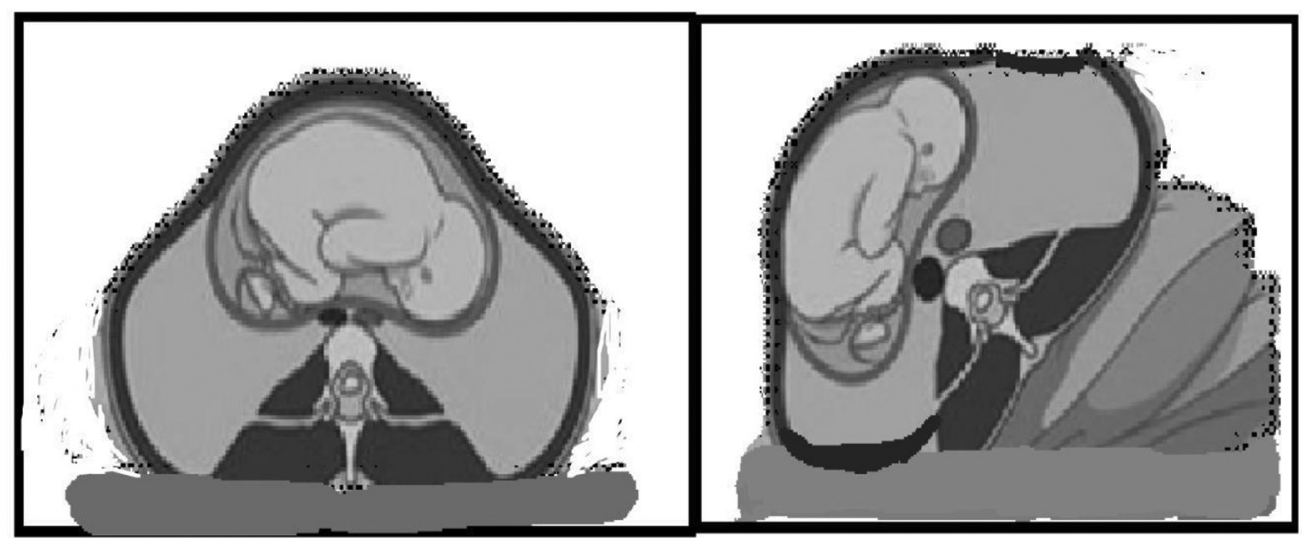

Aortocaval Compression by Gravid Uterus

Fig. 2 Left uterine tilt and its effect on aortocaval compression. hematocrit in the cases of hypovolemia and acute hemorrhage may be misinterpreted as physiologic anemia. Moreover, during hypovolemia, the blood flow tends to be shunted from the placenta and uterine circulation. Therefore, even though the parturient appears stable, the fetal blood flow is severely reduced and there can be a fetal compromise. Hence, it should be assessed without delay. It is essential to understand that there can be a blood loss of up to $35 \%$ of the blood volume in the absence of any overt signs of hemodynamic instability in a pregnant patient. Volume replacement should be commenced early and the intravenous fluid site should be secured above the level of the diaphragm for resuscitation.

There is an increase in oxygen consumption, tidal volume, cause a faster desaturation rate during periods of apnea in such patients. A decrease in functional residual capacity (FRC) is seen consequent to the expanding gravid uterus, elevation of the diaphragm, and reduction in thoracic volume. These changes result in more rapid development of hypoxemia necessitating the early establishment of oxygenation and ventilation. The consequences of hormonal changes of pregnancy such as upper airway edema, increased size of the breast, and weight gain can result in difficult as well as a delayed establishment of ventilation and intubation. Further, delayed gastric emptying caused by increased levels of progesterone increases the risk of aspiration during mask ventilation and intubation. The NPO (nil per oral) status is not always ensured as many patients are encountered after the consumption of a large meal. Hence, it is essential to maintain cricoid pressure during intubation. Starling forces narrow oncotic pressure-wedge pressure gradient increasing the risk of pulmonary edema. The $\mathrm{O}_{2}$ dissociation curve is shifted to the right; hence, a higher $\mathrm{O}_{2}$ partial pressure is required for adequate maternal oxygenation. Thus, pregnancy complicates airway management during resuscitation due to a potential full stomach, the risk of regurgitation, difficult ventilation, and intubation compounded by reduced safe apnea time and rapid desaturation. and minute ventilation in the pregnant state. These changes
Relief of Aortocaval Compression by Left Uterine tilt
The changes in pharmacokinetics may occur due to changes in plasma volume and increase in the glomerular filtration rate (GFR). However, there is no evidence that modification of the doses of medications used during resuscitation is required. ${ }^{25}$ Defibrillation is an essential component of cardiac resuscitation. The amniotic fluid transmits current. There could be a potential for fetal burns or adverse outcome if the current travels through the uterus. However, there are no studies on the fetal risk during maternal defibrillation. The risk of inducing fetal complications such as inducing fetal arrhythmias is small, ${ }^{26}$ and defibrillation is proven to be safe at all stages of pregnancy. There is a risk of electric arcing to monitors of the fetus, though low. Hence, it is advisable to remove internal or external fetal monitors before defibrillation in a pregnant woman.

\section{Management of Critically III Pregnant Patient}

The risk of cardiac arrest in a critically ill pregnant patient is very high. ${ }^{27,28}$ The hypotension is compounded by uterine obstruction of venous return that may even precipitate an arrest. The parturient patient should be placed in the full left-lateral position to relieve possible compression of the inferior vena cava. Oxygen should be administered early in the course as deterioration can be rapid. Maternal hypotension should be assessed and expeditiously treated as it not only affects the mother but also reduces placental perfusion. It is crucial to identify and address reversible causes of critical illness that contribute to deterioration as early as possible.

\section{Management of Cardiac Arrest}

The basics of basic life support (BLS) and ACLS protocol are the same as for nonpregnant victims of cardiac arrest, with a few necessary modifications. The physiologic and physical changes that accompany pregnancy mandate certain modifications during resuscitation of cardiac arrest in the parturient. 
The essential modifications are relieving aortocaval compression by the gravid uterus, performing rapid sequence intubation, and proceeding to timely perimortem cesarean delivery when indicated. A high-quality CPR is critical for survival. The algorithm for the management of cardiac arrest in pregnancy is shown in $\boldsymbol{- F i g} \mathbf{3}$.

\section{Recommended Modifications to Resuscitation Protocols Applied to Pregnant Patients}

The compression rate, depth, hand placement, backboard, and minimal interruptions in compressions are the same as nonpregnant adults. The gravid uterus causes elevation of the diaphragm; hence, the chest compressions should be performed slightly higher on the sternum than typically recommended. In late pregnancy, the gravid uterus acts like a binder for the abdomen that results in increased in intrathoracic pressure, reduced venous return, and reduction in the forward flow of blood through the abdominal aorta rendering successful resuscitation difficult. The best cardiac output that can be generated by external cardiac massage is only $30 \%$ of a nonpregnant patient. Besides, $10 \%$ of cardiac output is shunted to the gravid uterus. The increased oxygen requirements during pregnancy make the parturient more vulnerable to hypoxia.
Adequate left uterine displacement must be maintained during chest compressions. Large doses of $\alpha$-adrenergic may reduce uterine blood flow, but the use of these drugs is essential to restoring maternal circulation. If the efforts to resuscitate using chest compressions and medications are not successful, it is now recommended that cesarean section is performed within 4 to 5 minutes of the arrest. One must be vigilant about the time. Shorter intervals between the onset of maternal cardiac arrest and commencement of CPR, and shorter time taken to deliver the fetus once CPR commences, are associated with better maternal and fetal outcomes.

Management decisions depend on gestational age. For example, avoidance of aortocaval compression is essential after 15 weeks of gestation or when the fundal height is more than one handbreadth below the umbilicus. If brain death occurs before fetal maturation, continued somatic support of the mother can be done to extend the pregnancy until fetal maturation.

\section{Methods of Displacing Uterus}

The quality of CPR and resultant compression force and output are dependent on the position. The left-lateral tilt should be maximized to improve maternal hemodynamics during cardiac arrest. There are several practical issues regarding the left lateral tilt. It is not practical to apply a full lateral rotation

\section{CARDIAC ARREST IN PREGNANCY}

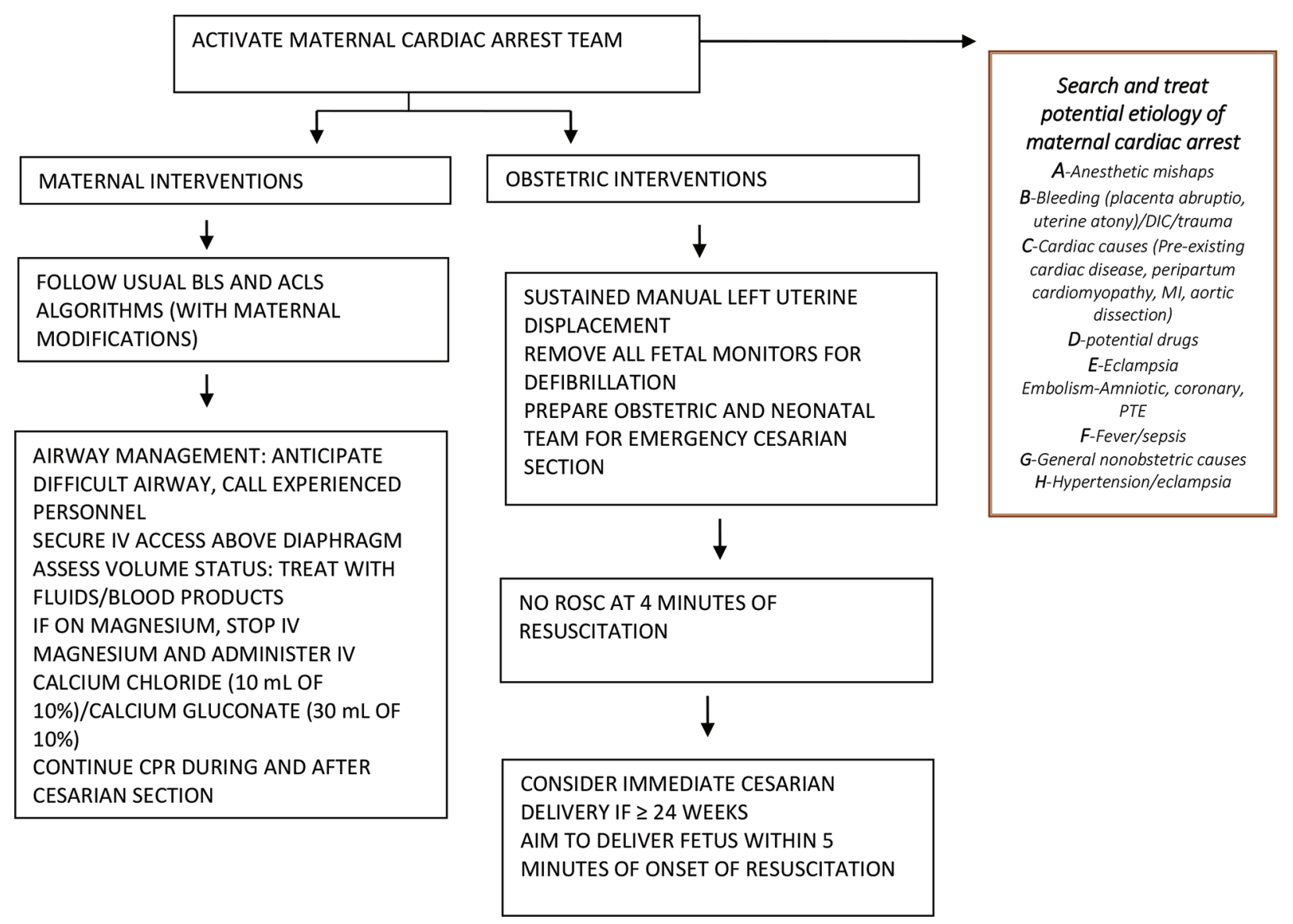

Fig. 3 Modifications for resuscitation of cardiac arrest in pregnancy. ACLS, advanced cardiac life support; BLS, basic life support; CPR, cardiopulmonary resuscitation; DIC, disseminated intravascular coagulation; MI, myocardial infarction; PTE, pulmonary thromboendarterectomy; ROSC, return of spontaneous circulation. 
during resuscitation. There is no improvement in maternal hemodynamic or fetal parameters with 10 to 20 degrees left-lateral tilt, and the patient may roll off the inclined plane if the slope exceeds 30 degrees. ${ }^{29,30}$ Moreover, the degree of tilt is difficult to estimate reliably. Hence, using a fixed, hard wedge with a predetermined angle is helpful. The Cardiff wedge provides both relief of aortocaval compression and a firm surface for performing cardiac compressions during resuscitation. When a wedge is not available, rolling up blankets to tilt the right hip or even a simple maneuver of tipping the patient on the bent knees of a kneeling rescuer ("human wedge") is useful. Although the left-lateral tilt position is vital to reduce aortocaval compression by gravid uterus, the chest compressions are less forceful than in the supine position. Manual left uterine displacement with the patient supine is comparable to left-lateral tilt in relieving aortocaval compression. ${ }^{31}$ Therefore, one can commence resuscitation with manual left uterine displacement in the supine position, using a two-handed technique from the patient's left side or the one-handed technique from the patient's right side depending on the location of the resuscitation team. ${ }^{32}$ If this is unsuccessful and an appropriate wedge is available, the patient should be placed in a left-lateral tilt of about 30 degrees using a firm wedge. If chest compressions remain inadequate even after lateral uterine displacement or left-lateral tilt, consider an immediate emergency cesarean section.

Arrhythmia-specific recommendations: Amiodarone $300 \mathrm{mg}$ rapid infusion followed by $150 \mathrm{mg}$ doses as needed is used to treat refractory Vfib, although fetal safety is questionable. ${ }^{33}$ Epinephrine is recommended over vasopressin. Atropine is administered only for bradycardia. Calcium is helpful to reverse magnesium toxicity. There is no need to alter drug doses or withhold emergency medications out of concerns for fetal teratogenicity.

\section{Perimortem Cesarean Section}

\section{Indications}

Emergency cesarean section in cardiac arrest resuscitation should be prepared in every cardiac arrest in a pregnant woman, with an evident gravid uterus large enough to cause maternal hemodynamic changes due to aortocaval compression regardless of fetal viability.

\section{Timing of Perimortem C-Section: 4-Minute Rule}

Perimortem C-section is lifesaving and should be performed at the site of arrest within 5 minutes. Maternal resuscitation efforts should be continued during the procedure. Early delivery decreases the risk of neurologic damage to the baby. If the fetus is viable, but the maternal prognosis is grave, and resuscitative efforts appear futile, it may be appropriate to perform an immediate emergency cesarean section without delay. Even if the emergency cesarean section cannot be performed by the 5-minute mark, it may be advisable to evacuate the uterus while the resuscitation continues (class IIb, LOE C). ${ }^{34}$ The emergency cesarean section team should be activated at the onset of maternal cardiac arrest if the gravid uterus is visible (class I, LOE B). An emergency cesarean section may be considered 4 minutes after onset of maternal cardiac arrest if there is no return of spontaneous circulation (class IIb, LOE C). ${ }^{34}$

\section{Expected Fetal and Maternal Outcomes of Perimortem Cesarean Section}

There is increasing use of PMCS due to the training (0.36/year before training to $1.6 /$ year after training, $p=0.01$ ). Although the timing of PMCS is the most crucial factor for both maternal and fetal outcomes, it was possible to perform PMCS within the recommended 5 minutes of commencing resuscitation in very few cases. ${ }^{35}$ The mean times to delivery in survivors $14.1 \pm 11$ minutes and $22 \pm 13$ minutes in nonsurvivors. ${ }^{36}$ There are reports of maternal survival of the mother with a PMCS performed up to 15 minutes after the onset of cardiac arrest. A study has shown that of the 12 women who underwent PMCS, (67\%) regained cardiac output, but only 2 mothers and 5 neonates survived. Maternal mortality rate was $83 \%$. A meta-analysis of maternal outcome after PMCS shows that $54.3 \%$ (51/94) of mothers survived to hospital discharge, $78.4 \%$ (40/51) with a cerebral performance category (CPC) of $1 / 2$. PMCS is shown to be beneficial to the mother in $31.7 \%$ of cases and was not harmful in any case. The maternal outcomes were better with in-hospital arrest (odds ratios [ORs] 5.17) and PMCD within 10 minutes of arrest (ORs 7.42). ${ }^{37}$ Therefore, a low admission threshold for pregnant women with cardiorespiratory decompensation is recommended. They should be admitted to a high dependency or intensive care unit with facilities for the performance of resuscitation and delivery within 10 minutes of arrest. The neonatal case fatality rate was $58 \% .^{37}$ The fetal outcomes depend on gestational age. At 24 to 25 weeks of gestation, delivery within 5 minutes of maternal arrest results in best fetal outcome, whereas at a gestational age of 30 weeks, every delivery that occurs after 5 minutes of maternal cardiac arrest is associated with good outcomes. There are reports of neonatal survival even when delivery occurred within 30 minutes after onset of maternal cardiac arrest. ${ }^{38}$

\section{Postcardiac Arrest Care}

The recommendation for therapeutic hypothermia in comatose pregnant patients after cardiac arrest is based on occasional case reports ${ }^{39}$ and current recommendations for the nonpregnant patients. Therapeutic hypothermia may be considered on an individual basis (class IIb, LOE C). ${ }^{34}$ The fetus should be continuously monitored for potential complications such as bradycardia.

\section{Extended Somatic Support after Brain Death during Pregnancy}

Brain death declaration is rarely done in pregnant patients. It is generally considered unethical and futile to support vital organs once brain death is diagnosed. However, in pregnancy, the issues of brain death are more complex. ${ }^{40}$ The issue of the appropriateness of continuing maternal somatic support to prolong gestation until delivery is debatable. The expected 
physiologic changes after brain death also pose challenges to successful organ system support and fetal well-being. Since 1979 , there have been only 43 cases of maternal brain death, and most of them had normal fetal outcomes. ${ }^{41}$

\section{Challenges of Resuscitation of Pregnant Patients in Resource-Constrained Countries}

Several pregnant patients have limited access to health care, and most deliveries continue to be performed by midwives with inadequate skills in resuscitation. It is important to identify high-risk patients for cardiac arrest and treat them in specialized centers. Physicians, paramedical staff, and midwives need to be trained in CPR and understand the modifications required for pregnant patients. Training of all the staff involved in the care of pregnant patients for basic and advanced obstetric life support is essential. There is still a big gap between the requirements and availability of advanced health care facilities and trained personnel to prevent and resuscitate cardiac arrests in pregnant patients. Maternal deaths continue to plague the resource-constrained countries such as India.

\section{Conclusion}

Although cardiopulmonary arrest rarely occurs in the pregnant woman, the knowledge of principles of resuscitation in a parturient in the health care team is vital to facilitate positive outcomes for both the mother and the fetus. The principles of the airway, breathing, and circulation used in cardiopulmonary arrest are similar to any other CPR; however, the team must be familiar with the modifications that must be made because of the physical and physiologic changes that occur during pregnancy. If resuscitation is not successful within 5 minutes of cardiac arrest, a cesarean delivery must be attempted if uterine size indicates gestational age of at least 20 weeks. A coordinated team consisting of obstetricians, anesthesiologists, neonatologists, and nursing staff must work in an organized fashion for efficient and successful resuscitation of the pregnant patient and good fetal outcomes.

\section{Conflict of Interest}

None declared.

\section{References}

1 Beckett VA, Knight M, Sharpe P. The CAPS study: incidence, management and outcomes of cardiac arrest in pregnancy in the UK: a prospective, descriptive study. BJOG 2017;124(9): 1374-1381

2 Creanga AA, Syverson C, Seed K, Callaghan WM. Pregnancyrelated mortality in the United States, 2011-2013. Obstet Gynecol 2017;130(2):366-373

3 Lipman S, Cohen S, Einav S, et al; Society for Obstetric Anesthesia and Perinatology. The Society for Obstetric Anesthesia and Perinatology consensus statement on the management of cardiac arrest in pregnancy. Anesth Analg 2014;118(5):1003-1016
4 McQUAID E, Leffert LR, Bateman BT. The role of the anesthesiologist in preventing severe maternal morbidity and mortality. Clin Obstet Gynecol 2018;61(2):372-386

5 Subbaiah M, Sharma V, Kumar S, et al. Heart disease in pregnancy: cardiac and obstetric outcomes. Arch Gynecol Obstet 2013;288(1):23-27

6 van Hagen IM, Thorne SA, Taha N, et al; ROPAC Investigators and EORP Team. Pregnancy outcomes in women with rheumatic mitral valve disease: results from the registry of pregnancy and cardiac disease. Circulation 2018;137(8):806-816

7 Honigberg MC, Scott NS. Pregnancy-associated myocardial infarction. Curr Treat Options Cardiovasc Med 2018;20(7):58

8 Kealey A. Coronary artery disease and myocardial infarction in pregnancy: a review of epidemiology, diagnosis, and medical and surgical management. Can J Cardiol 2010;26(6): 185-189

9 Burchill LJ, Lameijer H, Roos-Hesselink JW, et al. Pregnancy risks in women with pre-existing coronary artery disease, or following acute coronary syndrome. Heart 2015;101(7): 525-529

10 Furenäs E, Eriksson P, Wennerholm UB, Dellborg M. Effect of maternal age and cardiac disease severity on outcome of pregnancy in women with congenital heart disease. Int J Cardiol 2017;243:197-203

11 Kampman MA, Valente MA, van Melle JP, et al; ZAHARA II investigators. Cardiac adaption during pregnancy in women with congenital heart disease and healthy women. Heart 2016;102(16):1302-1308

12 Sliwa K, van Hagen IM, Budts W, et al; ROPAC investigators. Pulmonary hypertension and pregnancy outcomes: data from the Registry Of Pregnancy And Cardiac Disease (ROPAC) of the European Society of Cardiology. Eur J Heart Fail 2016;18(9):1119-1128

13 Pieper PG, Balci A, Aarnoudse JG, et al; ZAHARA II investigators. Uteroplacental blood flow, cardiac function, and pregnancy outcome in women with congenital heart disease. Circulation 2013;128(23):2478-2487

14 van Hagen IM, Boersma E, Johnson MR, et al; ROPAC investigators and EORP team. Global cardiac risk assessment in the Registry Of Pregnancy And Cardiac disease: results of a registry from the European Society of Cardiology. Eur J Heart Fail 2016;18(5):523-533

15 Ghulmiyyah L, Sibai B. Maternal mortality from preeclampsia/ eclampsia. Semin Perinatol 2012;36(1):56-59

16 Vigil-De Gracia P, Rojas-Suarez J, Ramos E, et al. Incidence of eclampsia with HELLP syndrome and associated mortality in Latin America. Int J Gynaecol Obstet 2015;129(3):219-222

17 Dado CD, Levinson AT, Bourjeily G. Pregnancy and pulmonary embolism. Clin Chest Med 2018;39(3):525-537

18 Oami T, Oshima T, Oku R, Nakanishi K. Successful treatment of pulmonary embolism-induced cardiac arrest by thrombolysis and targeted temperature management during pregnancy. Acute Med Surg 2018;5(3):292-295

19 Brennan MC, Moore LE. Pulmonary embolism and amniotic fluid embolism in pregnancy. Obstet Gynecol Clin North Am 2013;40(1):27-35

20 Benson MD. Amniotic fluid embolism mortality rate. J Obstet Gynaecol Res 2017;43(11):1714-1718

21 Lee PH, Shulman MS, Vellayappan U, Symes JF, Olenchock SA Jr. Surgical treatment of an amniotic fluid embolism with cardiopulmonary collapse. Ann Thorac Surg 2010;90(5): 1694-1696

22 Schlembach D, Helmer H, Henrich W, et al. Peripartum haemorrhage, diagnosis and therapy. Guideline of the DGGG, OEGGG and SGGG (S2k Level, AWMF Registry No.015/063, March 2016). Geburtshilfe Frauenheilkd 2018;78(4):382-399

23 Paternoster DM, Manganelli F, Fantinato S, Gerace P. Maternal complications from tocolytic treatment with 
ritodrine. Three cases of pulmonary edema. Minerva Ginecol 2004;56(5):491-492

24 Hawkins JL, Chang J, Palmer SK, Gibbs CP, Callaghan WM. Anesthesia-related maternal mortality in the United States: 1979-2002. Obstet Gynecol 2011;117(1):69-74

25 Nanson J, Elcock D, Williams M, Deakin CD. Do physiological changes in pregnancy change defibrillation energy requirements? Br J Anaesth 2001;87(2):237-239

26 Wang YC, Chen CH, Su HY, Yu MH. The impact of maternal cardioversion on fetal haemodynamics. Eur J Obstet Gynecol Reprod Biol 2006;126(2):268-269

27 Healy ME, Kozubal DE, Horn AE, Vilke GM, Chan TC, Ufberg JW. Care of the critically ill pregnant patient and perimortem cesarean delivery in the emergency department. J Emerg Med 2016;51(2):172-177

28 Adeniran AS, Bolaji BO, Fawole AA, Oyedepo OO. Predictors of maternal mortality among critically ill obstetric patients. Malawi Med J 2015;27(1):16-19

29 Fields JM, Catallo K, Au AK, et al. Resuscitation of the pregnant patient: what is the effect of patient positioning on inferior vena cava diameter? Resuscitation 2013;84(3):304-308

30 Ip JK, Campbell JP, Bushby D, Yentis SM. Cardiopulmonary resuscitation in the pregnant patient: a manikin-based evaluation of methods for producing lateral tilt. Anaesthesia 2013;68(7):694-699

31 Dohi S, Ichizuka K, Matsuoka R, Seo K, Nagatsuka M, Sekizawa A. Coronary perfusion pressure and compression quality in maternal cardiopulmonary resuscitation in supine and left-lateral tilt positions: A prospective, crossover study using mannequins and swine models. Eur J Obstet Gynecol Reprod Biol 2017;216:98-103

32 Jain V, Chari R, Maslovitz S, et al; Maternal Fetal Medicine Committee. Guidelines for the management of a pregnant trauma patient. J Obstet Gynaecol Can 2015;37(6):553-574
33 Gowda RM, Khan IA, Mehta NJ, Vasavada BC, Sacchi TJ. Cardiac arrhythmias in pregnancy: clinical and therapeutic considerations. Int J Cardiol 2003;88(2-3):129-133

34 Lavonas EJ, Drennan IR, Gabrielli A, et al. Part 10: special circumstances of resuscitation: 2015 American Heart Association guidelines update for cardiopulmonary resuscitation and emergency cardiovascular care. Circulation 2015;132 (18, Suppl 2):S501-S518

35 Smith A, Edwards S, Siassakos D. Effective team training to improve outcomes in maternal collapse and perimortem caesarean section. Resuscitation 2012;83(10):1183-1184

36 Dijkman A, Huisman CM, Smit M, et al. Cardiac arrest in pregnancy: increasing use of perimortem caesarean section due to emergency skills training? BJOG 2010;117(3):282-287

37 Einav S, Kaufman N, Sela HY. Maternal cardiac arrest and perimortem caesarean delivery: evidence or expert-based? Resuscitation 2012;83(10):1191-1200

38 Rose CH, Faksh A, Traynor KD, Cabrera D, Arendt KW, Brost $\mathrm{BC}$. Challenging the 4- to 5-minute rule: from perimortem cesarean to resuscitative hysterotomy. Am J Obstet Gynecol 2015;213(5):653-656, 653.e1

39 Rittenberger JC, Kelly E, Jang D, Greer K, Heffner A. Successful outcome utilizing hypothermia after cardiac arrest in pregnancy: a case report. Crit Care Med 2008;36(4):1354-1356

40 Gopčević A, Rode B, Vučić M, et al. Ethical and medical management of a pregnant woman with brain stem death resulting in delivery of a healthy child and organ donation. Int J Obstet Anesth 2017;32:82-86

41 Said A, Amer AJ, Masood UR, Dirar A, Faris C. A brain-dead pregnant woman with prolonged somatic support and successful neonatal outcome: A grand rounds case with a detailed review of literature and ethical considerations. Int J Crit Illn Inj Sci 2013;3(3):220-224 